\title{
Development and validation of an LC-MS/MS method for the determination of biogenic amines in wines and beers
}

\author{
Katarzyna Nalazek-Rudnicka ${ }^{1}$ (D) Andrzej Wasik ${ }^{1}$
}

Received: 1 March 2017 / Accepted: 2 May 2017/Published online: 11 July 2017

(c) The Author(s) 2017. This article is an open access publication

\begin{abstract}
Biogenic amines are group of organic, basic, nitrogenous compounds that naturally occur in plant, microorganism, and animal organisms. Biogenic amines are mainly produced through decarboxylation of amino acids. They are formed during manufacturing of some kind of food and beverages such as cheese, wine, or beer. Histamine, cadaverine, agmatine, tyramine, putrescine, and $\beta$-phenylethylamine are the most common biogenic amines found in wines and beers. This group of compounds can be toxic at high concentrations; therefore, their control is very important. Analysis of biogenic amines in alcoholic drinks (beers and wines) was carried out by HPLC-MS/MS after their derivatization with $p$-toluenesulfonyl chloride (tosyl chloride). The developed method has been applied for analysis of seventeen biogenic amines in twentyeight samples of lager beers and in twelve samples of different homemade wines (white grape, red grape, strawberry, chokeberry, black currant, plum, apple, raspberry, and quince). The developed method is sensitive and repeatable for majority of the analytes. It is versatile and can be used for the determination of biogenic amines in various alcoholic beverages.

Graphical abstract
\end{abstract}

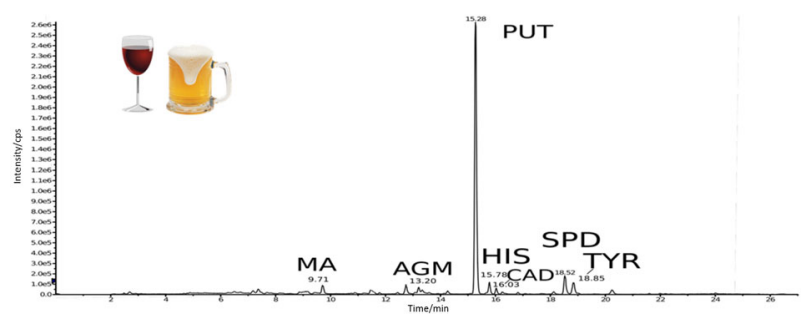

Katarzyna Nalazek-Rudnicka

katnalaz@ student.pg.gda.pl

1 Department of Analytical Chemistry, Faculty of Chemistry, Gdańsk University of Technology, ul. G. Narutowicza 11/12, 80-233 Gdańsk, Poland
Keywords Biogenic amines - Derivatization .

Tosyl chloride $\cdot$ High performance liquid chromatography · Mass spectrometry

\section{Introduction}

Biogenic amines (BA's) are nitrogenous organic bases, occurring mainly in fermented food and beverages as a result of free amino acids bacterial decarboxylation. This reaction involves the transformation of the amino acid to biogenic amine after removal of the carboxyl group by decarboxylase enzyme or transamination of aldehydes and ketones by amino acid transaminases. In non-fermented food, biogenic amines may also occur as a result of undesirable microbial activity but at lower concentrations [1-3].

Concentrations of biogenic amines in foods (meat, cheese, beer, etc.) can be an indicator of their freshness and/or hygienic quality [4-6]. Some authors propose food quality indexes, so-called biogenic amine indexes-BAI, based on the concentrations of selected biogenic amines (e.g., cadaverine, putrescine, histamine, spermidine, spermine, and tyramine) for this purpose. In case of beers, levels of specific biogenic amines may provide an information about quality and type of the fermentation process. According to the literature, the highest content of biogenic amines is typically found in beers from spontaneous fermentation, while in case of low fermentation, biogenic amines levels are up to 20 times lower [6].

Content of biogenic amines in foods and beverages may reach levels at which they become toxic and dangerous to human health, so their determination is extremely important. Although moderate doses of biogenic amines (around $50 \mathrm{mg} / \mathrm{kg}$ food) are harmless to most people, higher 
amounts can cause serious consequences [6]. High content of some biogenic amines such as histamine, tryptamine, tyramine, or $\beta$-phenylethylamine in food and beverages may result in a range of health problems. In case of histamine poisoning, the most common symptoms among patients are nausea, diarrhea, rash, hypotension, and headache, while 'overdose' of tyramine may cause effects such as hypertension or migraine. It is worth to note that the presence of some of polyamines, mainly cadaverine or putrescine, may increase the toxicity of histamine and tyramine. Furthermore, polyamines such as cadaverine, putrescine, spermine, and spermidine by reaction with nitrite lead to the formation of carcinogenic nitrosamines. Additionally, the consumption of food rich in tyramine or histamine and monoamino (MAOI) or diamino (DAOI) oxidase inhibitor drugs at the same time can lead to hypertensive crisis and allergic reactions, respectively [6-10].

It is suggested that in case of alcoholic beverages, toxic impact of biogenic amines is not connected with their high concentration in these beverages, but with the consumption of large quantities of beer or wine in a very short time. Additionally, alcohol can reduce the activity of mono- and diamino oxidases (MAO, DAO), thus preventing their distribution in the organism. What is more, acetaldehyde and antidepressant drugs can also inhibit activity of these enzymes [10, 11]. Due to the above-mentioned risks, controlling the content of biogenic amines in alcoholic beverages (beers or wines) is definitely important. Additionally, biogenic amines fingerprint can be used to verify the authenticity of wines or beers and can also be used to trace their origin or in case of beers - type of fermentation [12-14].

Determination of biogenic amines in food and beverages may be problematic because of the polar nature of these compounds, low levels of concentrations and complex sample matrix [15]. To determine biogenic amines in foods, a lot of analytical techniques like gas chromatography, thin layer chromatography, capillary electrophoresis and high performance liquid chromatography had been applied. Because of high sensitivity, resolution, great versatility, and relatively simple sample preparation, HPLC seems to be one of the most extensively used techniques [1, 15]. Additionally, multiple reaction monitoring mode HPLC-MS/MS provides highly sensitive and selective detection.

Determination of biogenic amines in their native state by HPLC is difficult, because of low sensitivity and due to the severe peak tailing. To improve chromatographic behavior of these compounds, many derivatization agents have been applied [1, 15].

The choice of derivatization agent depends on detector or chromatographic technique [2]. According to the literature, the most frequently chosen derivatization agents are dansyl chloride (DNS-Cl), dabsyl chloride (DBS-Cl) benzoyl chloride, 4-chloro-3,5- dinitrobenzotrifluoride (CNBF), $o$ phthalaldehyde (OPA), diethyl ethoxymethylenemalonate (DEEMM), 6-aminoquinolyl- $N$-hydroxysuccinimidyl carbamate (AQC), 9H-fluoroen-9-ylmethyl chloroformate (FMOC-Cl), or tosyl chloride (TSCl). It is worth noting that the use of tosyl chloride for derivatization of monoamines is not widely encountered. There are several publications where tosyl chloride was used as derivatization agent but in most cases it was used to derivatize polyamines [9, 16-18].

The purpose of the work described in this paper was to develop an LC-MS/MS method for determination of biogenic amines in alcoholic beverages (beers and wines) after their derivatization with tosyl chloride.

\section{Results and discussion}

To separate derivatives of fourteen biogenic amines, the gradient elution program was developed. The best results, in terms of the sensitivity and peak shape, were obtained when the mobile phase consisted of acetonitrile and water acidified with formic acid. Column temperature was set to $40^{\circ} \mathrm{C}$, in order to lower system's backpressure.

Derivatization conditions, such as derivatization time, temperature, volumes of sample and reagents, and concentration of tosyl chloride, were optimized in terms of repeatability and efficiency (sensitivity). It was observed that in case of some compounds under the study (e.g., tryptamine and agmatine) relatively long derivatization time $(120 \mathrm{~min})$ was required to improve repeatability. Additionally, it was also found that stopping the derivatization reaction with $1 \mathrm{M} \mathrm{HCl}$, as suggested Dziarkowska et al. [18], caused loss of repeatability-probably due to hydrolytic degradation of some tosyl derivatives (sulfonamides) at $\mathrm{pH}$ around 2.0.

Preliminary analyses of wine and beer samples were performed to select concentration ranges for six-point calibration curves. Since the content of some BA's (isopentylamine, putrescine, tyramine, and agmatine) in beer samples was much higher than in wine samples, it was necessary to prepare two sets of calibration solutions, covering low and high concentration ranges. The calibration curves were linear within the studied concentration ranges and were characterized by coefficients of determination higher than 0.99 (Table 1).

Repeatability of the method was estimated from triplicate analyses of wine (Table 2) and beer (Table 3) samples. For most samples, the coefficient of variation was relatively low (values were within criteria of acceptance; $C V<15 \%)$. 
Twenty-eight different commercially available beer and twelve different homemade wine samples were analyzed using the developed method. Out of seventeen biogenic amines being studied, fourteen were detected. Hexylamine, diethylamine, and propylamine were not detected in any of the analyzed wine nor beer samples.

\section{Homemade wines}

According to the literature, the most common biogenic amines in grape wines are tyramine, putrescine, cadaverine, 2-phenylethylamine, and histamine [19]. Analyses of our wine samples (Table 2) revealed that of all studied biogenic amines putrescine achieved the highest concentration levels (up to $3300 \mu \mathrm{g} / \mathrm{dm}^{3}$ ) followed by spermidine (up to $2600 \mu \mathrm{g}$ / $\mathrm{dm}^{3}$ ) and agmatine (up to $1160 \mu \mathrm{g} / \mathrm{dm}^{3}$ ). Eight other amines were also detected; however, at much lower $\left(<350 \mu \mathrm{g} / \mathrm{dm}^{3}\right)$ concentration levels. These included methylamine, dimethylamine, spermine, tyramine, $\quad \beta$-phenylethylamine, isopentylamine, histamine, and cadaverine. Histamine which may have adverse health effects was detected in majority of samples but at a rather low concentration levels (up to $125 \mu \mathrm{g}$ / $\mathrm{dm}^{3}$ in red grape wine). The highest amounts of histamine were found in two red grape wines followed by white grape wine, apple, and raspberry wines. A typical chromatogram obtained after derivatisation and HPLC-MS/MS analysis of a wine sample is shown in Fig. 1.

The high content of putrescine in itself is not harmful, but may increase the toxicity of accompanying histamine or tyramine. Such effect can be anticipated in case of grape wines which contain the highest amounts of biogenic amines among the tested samples.

Agmatine and cadaverine were detected only in red grape wine samples. In general, grape wines contain significantly higher amounts of biogenic amines than wines produced from other fruits (Fig. 2).

\section{Beer}

The survey of the literature data reveals that the most dominant biogenic amines in beers are agmatine and putrescine. Additionally, histamine, tyramine, and

Table 1 Calibration data for biogenic amines under the study

\begin{tabular}{|c|c|c|c|c|c|c|}
\hline Compound & Calibration range $/ \mu \mathrm{g} \mathrm{dm}^{-3}$ & Slope & Intercept & $R^{2}$ & $\begin{array}{l}\text { LOD/ } \\
\mu \mathrm{g} \mathrm{dm}^{-3}\end{array}$ & $\begin{array}{l}L O Q / \\
\mu \mathrm{g} \mathrm{dm}^{-3}\end{array}$ \\
\hline Methylamine & $46-830$ & $1.613 \mathrm{E}+06$ & $2.243 \mathrm{E}+04$ & 0.9989 & 0.023 & 0.075 \\
\hline Dimethylamine & $4.7-84$ & $2.743 \mathrm{E}+04$ & $4.158 \mathrm{E}+04$ & 0.9983 & 2.9 & 9.6 \\
\hline Propylamine & $110-1950$ & $1.833 \mathrm{E}+03$ & $5.938 \mathrm{E}+04$ & 0.9998 & 24 & 78 \\
\hline Butylamine & $3.6-65$ & $1.226 \mathrm{E}+03$ & $3.688 \mathrm{E}+03$ & 0.9976 & 3.4 & 11 \\
\hline Isobutylamine & $3.3-59$ & $1.149 \mathrm{E}+03$ & $3.976 \mathrm{E}+03$ & 0.9998 & 0.82 & 2.8 \\
\hline Diethylamine & $1.6-29$ & $3.158 \mathrm{E}+03$ & $1.506 \mathrm{E}+04$ & 0.9997 & 0.44 & 1.4 \\
\hline 2-Phenylethylamine & $7.3-130$ & $1.470 \mathrm{E}+03$ & $8.361 \mathrm{E}+03$ & 0.9978 & 5.4 & 18 \\
\hline \multirow[t]{2}{*}{ Isopentylamine } & $3.2-58$ & $6.278 \mathrm{E}+02$ & $-1.137 \mathrm{E}+03$ & 0.9982 & 2.7 & 9.0 \\
\hline & $120-2100$ & $5.695 \mathrm{E}+02$ & $8.476 \mathrm{E}+02$ & 0.9957 & - & - \\
\hline \multirow[t]{2}{*}{ Tryptamine } & $50-910$ & $6.546 \mathrm{E}+03$ & $-1.934 \mathrm{E}+05$ & 0.9998 & 11 & 36 \\
\hline & $400-7300$ & $4.758 \mathrm{E}+03$ & $1.121 \mathrm{E}+06$ & 0.9956 & - & - \\
\hline \multirow[t]{2}{*}{ Cadaverine } & $41-730$ & $6.594 \mathrm{E}+03$ & $-1.685 E+05$ & 0.9996 & 10 & 34 \\
\hline & $130-2300$ & $4.975 \mathrm{E}+03$ & $3.702 \mathrm{E}+05$ & 0.9989 & - & - \\
\hline \multirow[t]{2}{*}{ Putrescine } & $20-370$ & $5.545 \mathrm{E}+03$ & $4.629 \mathrm{E}+04$ & 0.9994 & 7.3 & 24 \\
\hline & 730-13,000 & $3.613 \mathrm{E}+03$ & $8.016 \mathrm{E}+06$ & 0.9984 & - & - \\
\hline \multirow[t]{2}{*}{ Spermidine } & 19-340 & $8.481 \mathrm{E}+03$ & $-6.489 E+04$ & 0.9999 & 3.6 & 12 \\
\hline & $200-3600$ & $8.298 \mathrm{E}+03$ & $8.260 \mathrm{E}+04$ & 0.9964 & - & - \\
\hline Spermine & $24-440$ & $5.914 \mathrm{E}+02$ & $2.657 \mathrm{E}+03$ & 0.9989 & 12 & 40 \\
\hline \multirow[t]{2}{*}{ Tyramine } & $19-340$ & $2.513 \mathrm{E}+03$ & $2.019 \mathrm{E}+04$ & 0.9979 & 11 & 36 \\
\hline & $200-3600$ & $1.565 \mathrm{E}+03$ & $4.527 \mathrm{E}+05$ & 0.9935 & - & - \\
\hline Histamine & $9.4-170$ & $7.327 \mathrm{E}+03$ & $2.689 \mathrm{E}+04$ & 0.9993 & 3.8 & 13 \\
\hline Hexylamine & $100-1800$ & $1.155 \mathrm{E}+03$ & $3.688 \mathrm{E}+04$ & 0.9995 & 36 & 120 \\
\hline \multirow[t]{2}{*}{ Agmatine } & $100-1800$ & $8.766 \mathrm{E}+02$ & $1.188 \mathrm{E}+05$ & 0.9973 & 83 & 270 \\
\hline & $2400-44,000$ & $5.955 \mathrm{E}+02$ & $1.161 \mathrm{E}+06$ & 0.9984 & - & - \\
\hline
\end{tabular}


Table 2 Content of biogenic amines in analyzed wines $\left(\mu \mathrm{g} / \mathrm{dm}^{3} \pm\right.$ standard deviation, $\left.n=3\right)$

\begin{tabular}{|c|c|c|c|c|c|c|}
\hline Wine & DMA & MA & PUT & SPD & SPM & TYR \\
\hline Grape_1 & $34.18 \pm 0.80$ & $292.2 \pm 5.6$ & $3090 \pm 89$ & $338 \pm 32$ & N/A & $232 \pm 16$ \\
\hline Grape_2 & $35.8 \pm 2.1$ & $282 \pm 21$ & $3333 \pm 218$ & $382 \pm 18$ & N/A & $203.9 \pm 9.8$ \\
\hline Grape (white) & N/A & $170.6 \pm 7.0$ & $1697 \pm 77$ & $2592 \pm 12$ & $346.0 \pm 3.5$ & N/A \\
\hline Black currant_1 & $16.69 \pm 0.83$ & $264 \pm 25$ & N/A & N/A & N/A & N/A \\
\hline Black currant_2 & N/A & N/A & N/A & N/A & N/A & N/A \\
\hline Plum_1 & $13.07 \pm 0.21$ & $231 \pm 19$ & $1125 \pm 15$ & N/A & N/A & $61.0 \pm 3.4$ \\
\hline Plum_2 & N/A & $128.1 \pm 6.4$ & N/A & N/A & N/A & N/A \\
\hline Chokeberry & N/A & N/A & N/A & N/A & N/A & N/A \\
\hline Quince & N/A & N/A & $493.5 \pm 6.9$ & $73.7 \pm 6.4$ & N/A & $32.4 \pm 1.3$ \\
\hline Raspberry & $28.03 \pm 0.27$ & $113.6 \pm 6.1$ & $71.9 \pm 9.5$ & $610 \pm 46$ & N/A & N/A \\
\hline Apple & $69.8954 \pm 0.0015$ & $232.1 \pm 4.9$ & N/A & $106.4 \pm 8.7$ & N/A & N/A \\
\hline Strawberry & $11.63 \pm 0.31$ & $292 \pm 21$ & N/A & $39.8 \pm 1.8$ & N/A & N/A \\
\hline $\mathrm{CV}^{\mathrm{a}}[\%]$ & $0.0022-5.9$ & $0.99-9.6$ & $1.4-13.3$ & $0.45-9.6$ & 1.0 & $4.0-6.7$ \\
\hline Wine & PHA & isoPEA & \multicolumn{2}{|c|}{ HIS } & AGM & $\mathrm{CAD}$ \\
\hline Grape_1 & $84.0 \pm 7.4$ & $53.7 \pm 1.5$ & \multicolumn{2}{|c|}{$124.0 \pm 7.6$} & $1031 \pm 40$ & $104 \pm 12$ \\
\hline Grape_2 & $73.1 \pm 2.4$ & $53.0 \pm 4.4$ & \multicolumn{2}{|c|}{$125.3 \pm 4.7$} & $1157 \pm 16$ & $107.1 \pm 8.9$ \\
\hline Grape (white) & $16.3 \pm 1.1$ & $132.1 \pm 4.3$ & \multicolumn{2}{|c|}{$55.9 \pm 1.5$} & N/A & N/A \\
\hline Black currant_1 & N/A & $35.26 \pm 0.23$ & \multicolumn{2}{|c|}{$21.96 \pm 0.90$} & N/A & N/A \\
\hline Black currant_2 & N/A & N/A & \multicolumn{2}{|c|}{$15.7 \pm 1.9$} & N/A & N/A \\
\hline Plum_1 & $37.3 \pm 2.1$ & N/A & \multicolumn{2}{|c|}{ N/A } & N/A & N/A \\
\hline Plum_2 & N/A & N/A & \multicolumn{2}{|c|}{$15.7 \pm 1.7$} & N/A & N/A \\
\hline Chokeberry & N/A & N/A & \multicolumn{2}{|c|}{ N/A } & N/A & N/A \\
\hline Quince & N/A & N/A & \multicolumn{2}{|c|}{ N/A } & N/A & N/A \\
\hline Raspberry & N/A & N/A & \multicolumn{2}{|c|}{$42.0 \pm 1.0$} & N/A & N/A \\
\hline Apple & $31.2 \pm 3.2$ & $25.7 \pm 2.6$ & \multicolumn{2}{|c|}{$45.62 \pm 0.92$} & N/A & N/A \\
\hline Strawberry & N/A & N/A & \multicolumn{2}{|c|}{ N/A } & N/A & N/A \\
\hline $\mathrm{CV}^{\mathrm{a}}[\%]$ & $3.3-10.3$ & $0.64-10.1$ & \multicolumn{2}{|c|}{$2.4-11.9$} & $1.4-3.9$ & $8.3-11.2$ \\
\hline
\end{tabular}

N/A not available

${ }^{\text {a }}$ Coefficient of variation $\mathrm{CV}=\mathrm{RSD} / \mathrm{X}_{\text {śr }}[\%]$

cadaverine also often found in beers, of which tyramine and cadaverine show the greatest fluctuations [20].

Our study partially confirms these findings (Table 3 ). Total content of biogenic amines in tested samples (Fig. 3) varied from 4225 to $38,510 \mu \mathrm{g} / \mathrm{dm}^{3}$ (median $15,875 \mu \mathrm{g}$ ) $\mathrm{dm}^{3}$ ). The dominant biogenic amines in analyzed beers, in terms of content, were: agmatine (median $7890 \mu \mathrm{g} / \mathrm{dm}^{3}$ ), putrescine (median $6030 \mu \mathrm{g} / \mathrm{dm}^{3}$ ), and tyramine (median $755 \mu \mathrm{g} / \mathrm{dm}^{3}$ ). However, putrescine was found in all analyzed samples while agmatine and tyramine only in some of them (in 61 and $89 \%$ of samples, respectively).

Other frequently found amines were: tryptamine $(100 \%$ of samples, median $170 \mu \mathrm{g} / \mathrm{dm}^{3}$ ), cadaverine $(93 \%$ of samples, median $330 \mu \mathrm{g} / \mathrm{dm}^{3}$ ), and spermidine ( $89 \%$ of samples, median $485 \mu \mathrm{g} / \mathrm{dm}^{3}$ ). Histamine was found only in $32 \%$ of samples at relatively low concentration levels (median $65 \mu \mathrm{g} / \mathrm{dm}^{3}$ ).

An interesting observation has been made during the data analysis. Two samples of the same brand name beer sold in identical containers (Table 3, samples pale lager $\mathrm{D}$ and pale lager J3) drastically differed in the content of tyramine. Sample pale lager J3 contained almost $13,000 \mu \mathrm{g} / \mathrm{dm}^{3}$ of tyramine while supposedly the same beer (sample pale lager D), taken from another can, contained only around $375 \mu \mathrm{g} /$ $\mathrm{dm}^{3}$ of this amine. Closer inspection of the two suspected containers revealed that these beers were produced in different places. It seems that the production plant may leave some kind of "amine signature" which could be used to trace the origin of the product. Verification of this hypothesis calls for more in-depth chemometric data analysis, however. 
Table 3 Content of biogenic amines in analyzed beers $\left(\mu \mathrm{g} / \mathrm{dm}^{3} \pm\right.$ standard deviation, $\left.n=3\right)$

\begin{tabular}{|c|c|c|c|c|c|c|}
\hline Beer & MA & BUA & isoBUA & isoPEA & PUT & SPD \\
\hline Pale lager A & $78 \pm 11$ & $11.3 \pm 1.6$ & $5.76 \pm 0.80$ & N/A & $5325 \pm 241$ & $192.7 \pm 5.6$ \\
\hline Premium lager A & $128 \pm 14$ & N/A & $8.62 \pm 0.77$ & $792.9 \pm 7.1$ & $7158 \pm 653$ & $739 \pm 81$ \\
\hline Strong pale lager A1 & $105.4 \pm 6.8$ & N/A & $7.96 \pm 0.35$ & $284 \pm 11$ & $6592 \pm 126$ & $367.2 \pm 5.4$ \\
\hline Strong pale lager A2 & $158.4 \pm 8.9$ & N/A & $9.36 \pm 0.84$ & $766 \pm 25$ & $8000 \pm 109$ & $781 \pm 51$ \\
\hline Standard american lager B & $168.6 \pm 6.5$ & $9.5 \pm 1.1$ & $11.3 \pm 1.1$ & $1043 \pm 30$ & $7858 \pm 101$ & $218 \pm 29$ \\
\hline Strong pale lager $\mathrm{C}$ & $107.7 \pm 6.0$ & $8.616 \pm 0.067$ & $10.362 \pm 0.067$ & $216.9 \pm 6.5$ & $6425 \pm 25$ & $381 \pm 56$ \\
\hline Standard american lager $\mathrm{C}$ & $84.6 \pm 9.2$ & N/A & $6.61 \pm 0.35$ & N/A & $4875 \pm 222$ & $271.8 \pm 8.3$ \\
\hline Pale lager $\mathrm{C} 1$ & N/A & N/A & $7.88 \pm 0.42$ & N/A & $5908 \pm 184$ & $245 \pm 20$ \\
\hline Pale lager $\mathrm{C} 2$ & $85.7 \pm 6.4$ & N/A & $5.70 \pm 0.42$ & N/A & $5667 \pm 88$ & $260 \pm 18$ \\
\hline Pale lager D & $87.4 \pm 4.0$ & N/A & $6.73 \pm 0.55$ & $293.9 \pm 4.4$ & $4567 \pm 138$ & $105 \pm 11$ \\
\hline Strong pale lager D & N/A & N/A & $8.5 \pm 1.0$ & N/A & $4858 \pm 250$ & N/A \\
\hline Pale lager E & $84.7 \pm 2.4$ & N.A & $7.95 \pm 0.54$ & $203.4 \pm 5.3$ & $5958 \pm 142$ & $444 \pm 16$ \\
\hline Strong pale lager $\mathrm{F}$ & $170.4 \pm 2.0$ & $13.52 \pm 0.80$ & $16.4 \pm 2.0$ & $172 \pm 10$ & $6475 \pm 87$ & $428 \pm 16$ \\
\hline Premium lager $\mathrm{F}$ & $198 \pm 15$ & $8.37 \pm 0.67$ & $10.02 \pm 0.83$ & $435 \pm 19$ & $6608 \pm 270$ & $638.5 \pm 6.3$ \\
\hline Pale lager F1 & $159.6 \pm 9.7$ & N/A & $11.31 \pm 0.12$ & $756 \pm 66$ & $7167 \pm 76$ & $721 \pm 82$ \\
\hline Pale lager F2 & $225 \pm 11$ & $11.7 \pm 1.3$ & $13.5 \pm 1.3$ & $560 \pm 46$ & $7300 \pm 307$ & $838 \pm 59$ \\
\hline Premium american lager $G$ & N/A & N/A & $4.429 \pm 0.094$ & N/A & $4725 \pm 413$ & $910 \pm 90$ \\
\hline Pale lager G1 & N/A & N/A & N/A & N/A & $3917 \pm 227$ & $653 \pm 58$ \\
\hline Pale lager G2 & $112.4 \pm 9.3$ & N/A & $5.55 \pm 0.12$ & N/A & $6100 \pm 132$ & $1408 \pm 76$ \\
\hline Premium lager $\mathrm{H}$ & $124 \pm 21$ & N/A & $9.36 \pm 0.90$ & N/A & $6750 \pm 71$ & $489 \pm 22$ \\
\hline Strong pale lager I & $357 \pm 19$ & N/A & N/A & N/A & $4550 \pm 205$ & N/A \\
\hline Pale lager I & $193 \pm 16$ & N/A & N/A & N/A & $3700 \pm 427$ & N/A \\
\hline Non alcoholic lager $\mathbf{J}$ & N/A & N/A & N/A & N/A & $3588 \pm 371$ & $739 \pm 74$ \\
\hline Witbier $\mathbf{J}$ & $92.9 \pm 5.8$ & $14.09 \pm 0.79$ & $4.262 \pm 0.047$ & $742.9 \pm 7.1$ & $5958 \pm 29$ & $222.2 \pm 2.5$ \\
\hline Pale lager J1 & $168 \pm 13$ & N/A & N/A & $925 \pm 10$ & $7417 \pm 146$ & $1301 \pm 106$ \\
\hline Pale lager J2 & $108 \pm 11$ & N/A & $5.846 \pm 0.071$ & $179.9 \pm 7.1$ & $6867 \pm 535$ & $400.37 \pm 0.88$ \\
\hline Pale lager J3 & N/A & N/A & $5.17 \pm 0.55$ & $463 \pm 20$ & $4542 \pm 138$ & $483 \pm 22$ \\
\hline Pale lager $\mathrm{K}$ & $186 \pm 12$ & $11.9 \pm 1.1$ & $13.7 \pm 1.1$ & $466 \pm 38$ & $8242 \pm 406$ & $1089 \pm 82$ \\
\hline $\mathrm{CV} / \%$ & $1.2-14.5$ & $0.77-14$ & $0.64-13.9$ & $0.89-8.7$ & $0.39-11.5$ & $0.22-14.6$ \\
\hline Beer & SPM & TYR & TRP & HIS & AGM & CAD \\
\hline Pale lager A & $178 \pm 19$ & $1240 \pm 74$ & $157.7 \pm 5.9$ & N/A & N/A & $238 \pm 21$ \\
\hline Premium lager A & N/A & $905 \pm 61$ & $216 \pm 30$ & $66.1 \pm 4.1$ & $7257 \pm 498$ & $494 \pm 27$ \\
\hline Strong pale lager A1 & $187 \pm 16$ & $816 \pm 93$ & $213 \pm 11$ & $54.5 \pm 2.1$ & $5983 \pm 354$ & $303 \pm 20$ \\
\hline Strong pale lager A2 & $181.0 \pm 9.6$ & $1274 \pm 164$ & $235.2 \pm 6.4$ & $82.3 \pm 2.8$ & $7651 \pm 209$ & $665 \pm 39$ \\
\hline Standard american lager B & N/A & $1413 \pm 195$ & $4239 \pm 151$ & $83.9 \pm 4.9$ & $11,509 \pm 509$ & $1089 \pm 47$ \\
\hline Strong pale lager $\mathrm{C}$ & $239 \pm 12$ & $757 \pm 58$ & $195.7 \pm 6.5$ & $70.2 \pm 9.1$ & N/A & $316 \pm 36$ \\
\hline Standard american lager $\mathrm{C}$ & N/A & N/A & $151.2 \pm 9.8$ & N/A & N/A & $428 \pm 26$ \\
\hline Pale lager $\mathrm{C} 1$ & $218.5 \pm 7.9$ & $791 \pm 60$ & $191 \pm 11$ & $66.9 \pm 6.9$ & N/A & $390 \pm 18$ \\
\hline Pale lager $\mathrm{C} 2$ & $214 \pm 13$ & N/A & $176.76 \pm 0.95$ & N/A & N/A & $185.2 \pm 8.1$ \\
\hline Pale lager D & N/A & N/A & $149.4 \pm 8.8$ & N/A & $6659 \pm 473$ & $174.5 \pm 5.3$ \\
\hline Strong pale lager D & N/A & N/A & $173 \pm 10$ & N/A & N/A & $210 \pm 24$ \\
\hline Pale lager E & N/A & N/A & $163.0 \pm 5.4$ & N/A & $5831 \pm 186$ & $285 \pm 27$ \\
\hline Strong pale lager $\mathrm{F}$ & $202 \pm 15$ & N/A & $191.3 \pm 9.9$ & N/A & $11,274 \pm 635$ & $291 \pm 14$ \\
\hline Premium lager $\mathrm{F}$ & $194.7 \pm 7.1$ & N/A & $157 \pm 11$ & N/A & $10,111 \pm 552$ & $341 \pm 10$ \\
\hline Pale lager F1 & $218.3 \pm 3.2$ & N/A & $161.3 \pm 8.5$ & N/A & $6729 \pm 433$ & $413 \pm 33$ \\
\hline Pale lager F2 & $233.8 \pm 4.4$ & N/A & $188 \pm 11$ & N/A & $8385 \pm 283$ & $361 \pm 18$ \\
\hline Premium american lager $G$ & $210 \pm 17$ & N/A & $137 \pm 12$ & N/A & $5496 \pm 199$ & N/A \\
\hline
\end{tabular}


Table 3 continued

\begin{tabular}{|c|c|c|c|c|c|c|}
\hline Beer & SPM & TYR & TRP & HIS & AGM & CAD \\
\hline Pale lager G1 & $152 \pm 10$ & N/A & $152.1 \pm 3.8$ & N/A & $5543 \pm 634$ & $177 \pm 16$ \\
\hline Pale lager G2 & $163 \pm 14$ & N/A & $171 \pm 13$ & N/A & $11,332 \pm 443$ & $219 \pm 15$ \\
\hline Premium lager $\mathrm{H}$ & N/A & N/A & $206 \pm 14$ & N/A & $9503 \pm 311$ & $372.5 \pm 2.8$ \\
\hline Strong pale lager I & N/A & N/A & $228 \pm 23$ & N/A & N/A & $463 \pm 43$ \\
\hline Pale lager I & N/A & N/A & $129.1 \pm 8.3$ & N/A & N/A & $203.2 \pm 1.2$ \\
\hline Non alcoholic lager $\mathrm{J}$ & N/A & $1720 \pm 85$ & $102.6 \pm 8.4$ & N/A & N/A & N/A \\
\hline Witbier $\mathbf{J}$ & N/A & $12,773 \pm 214$ & $140.4 \pm 2.9$ & $93.3 \pm 2.6$ & N/A & $553 \pm 29$ \\
\hline Pale lager J1 & $239 \pm 14$ & $16,800 \pm 140$ & $253 \pm 11$ & $50.86 \pm 0.18$ & $11,009 \pm 374$ & $348.5 \pm 5.7$ \\
\hline Pale lager $\mathbf{J} 2$ & $178 \pm 13$ & $897 \pm 44$ & $176 \pm 12$ & N/A & $7892 \pm 975$ & $234 \pm 20$ \\
\hline Pale lager J3 & N/A & $12,707 \pm 133$ & $134 \pm 12$ & N/A & N/A & $181.5 \pm 1.4$ \\
\hline Pale lager $\mathrm{K}$ & $213 \pm 14$ & $1405 \pm 182$ & $319 \pm 10$ & $48.6 \pm 5.4$ & $9847 \pm 643$ & $479 \pm 47$ \\
\hline $\mathrm{CV} / \%$ & $1.5-10.4$ & $0.83-13.8$ & $0.54-13.5$ & $0.35-13$ & $2.7-12.3$ & $0.57-11.5$ \\
\hline
\end{tabular}

N/A not available

$C$ coefficient of variation $\mathrm{CV}=\mathrm{RSD} / \mathrm{X}_{\text {ś }}[\%]$

- TIC of +MRM (32 pairs): from Sample I (winogrono_1.1) of WINO_27_06.wiff (Turbo Spray)

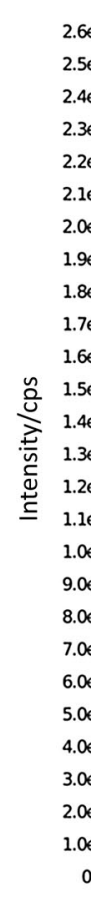

.

$19 \mathrm{e} 6$

$1.6 \mathrm{e}$

$1.5 \mathrm{e}$

1.4

$1.3 \mathrm{e}$

$1.1 \mathrm{e} 6$

oes-

Des

MA

-

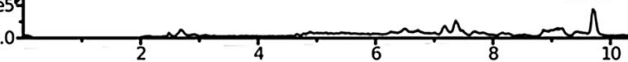

5.28

PUT

SPD

AGM

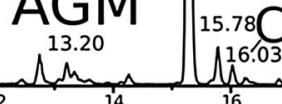

Time/min

Fig. 1 Example of total ion chromatogram obtained after analysis of red grape wine. $M A$ methylamine, AGM agmatine, PUT putrescine, $H I S$ histamine, $C A D$ cadaverine, SPD spermidine, TYR tyramine

\section{Conclusion}

A convenient method for the determination of seventeen biogenic amines in beers and wines was developed. Use of tandem mass spectrometric detection resulted in high sensitivity (LOQs at $\mu \mathrm{g} / \mathrm{dm}^{3}$ level) and selectivity. The method is characterized by satisfactory accuracy $(75-120 \%)$ and repeatability $(C V<15 \%)$. Derivatization procedure relies on the readily available tosyl chloride and does not require any specialized equipment. The method 
Fig. 2 Total concentration of biogenic amines in wines $/ \mu \mathrm{g} /$ $\mathrm{dm}^{3}$
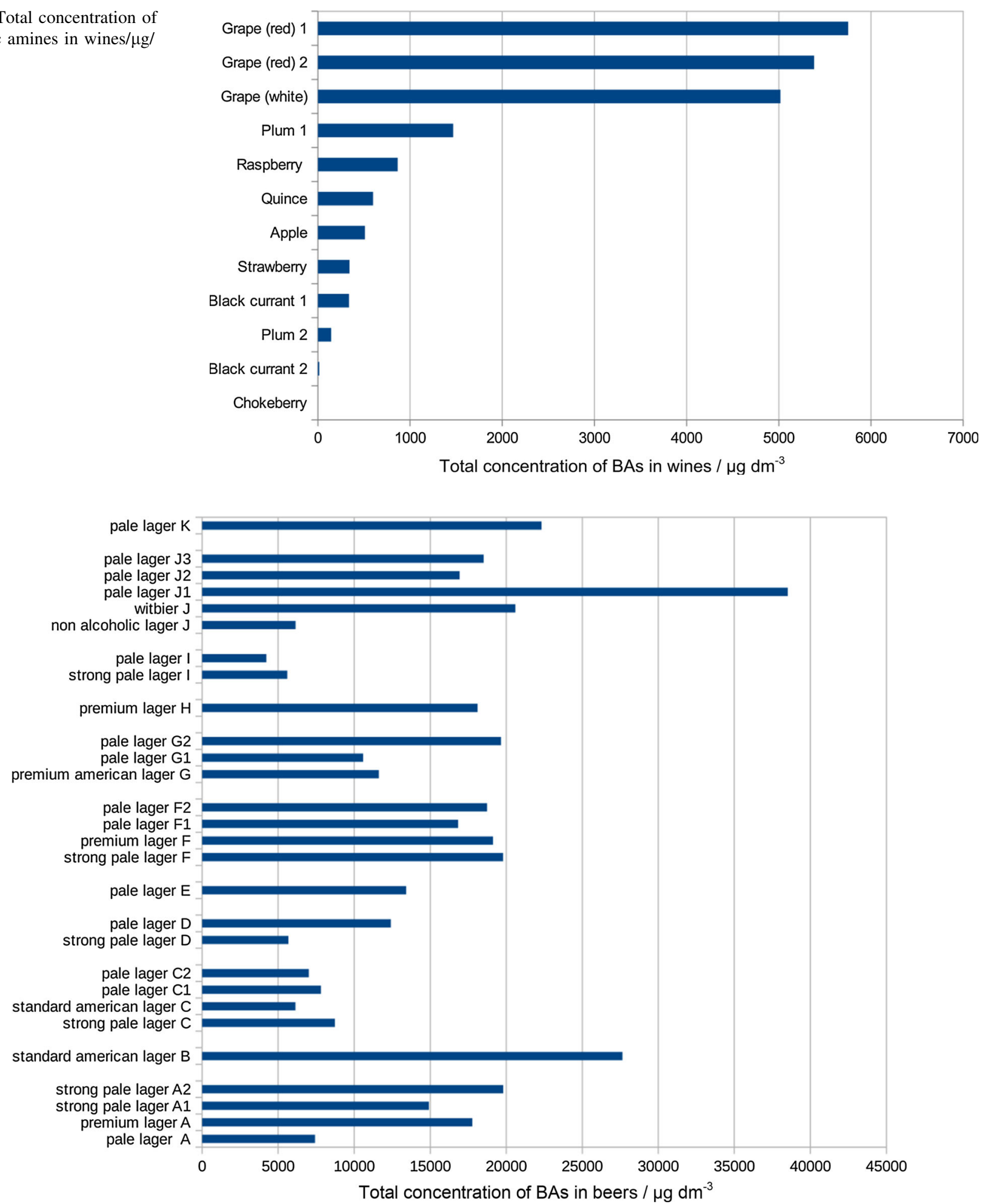

Fig. 3 Total concentration of biogenic amines in beers $/ \mu \mathrm{g} / \mathrm{dm}^{3}$

was successfully used for the determination of biogenic amines in the number of homemade wine and commercially available beer samples.
In general, grape wines contain around three times lower amounts of biogenic amines than beers. The amount of these amines in wines made from other fruits 
Fig. 4 Chemical structures of biogenic amines under the study

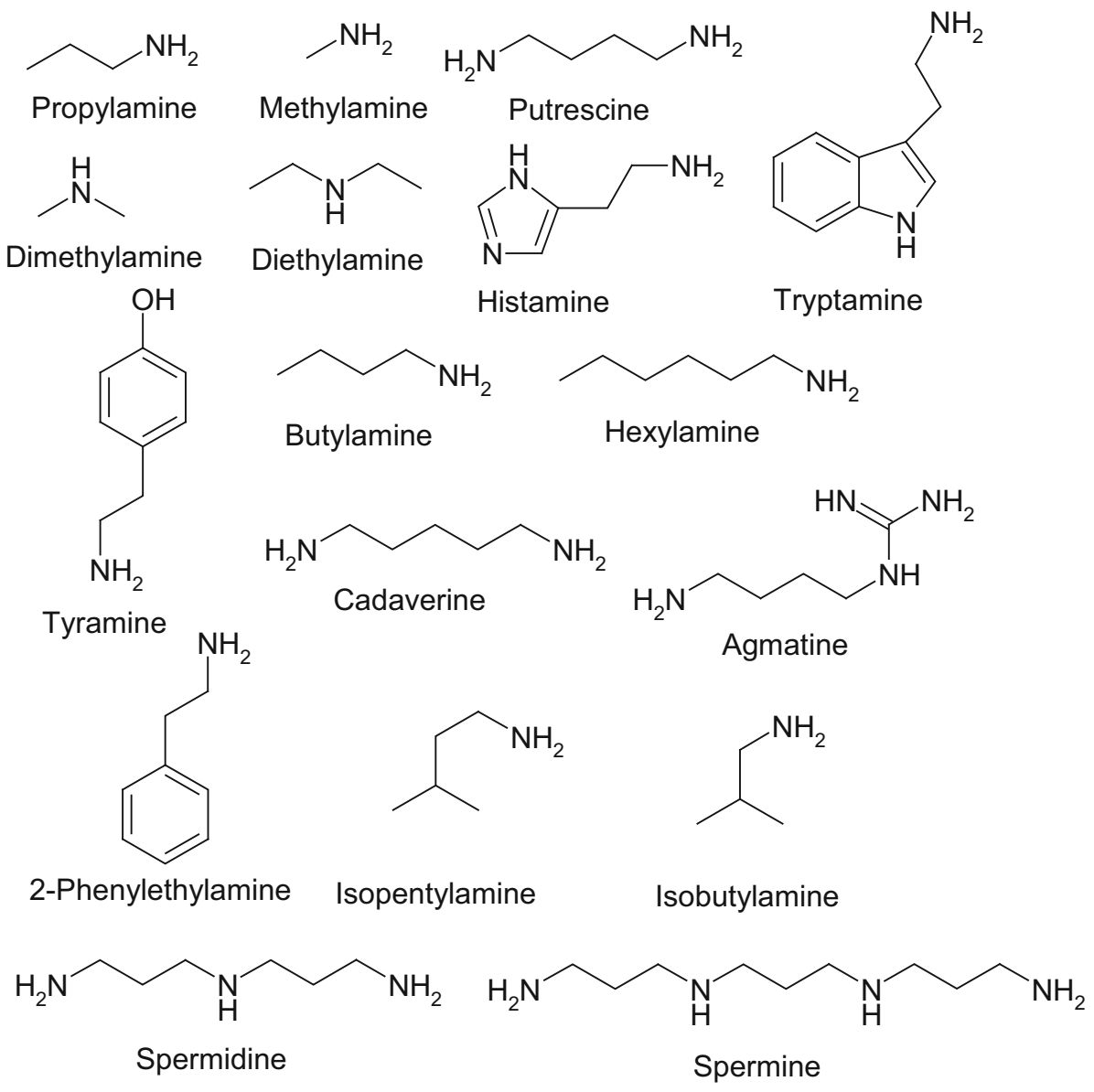

is even lower and strongly dependent on the type of fruit. Such information may be of a great value for those under treatment with monoamine oxidase inhibitors. Diverse levels of biogenic amines found in wines and beers may serve as their origin and originality markers. Such meta-analysis can be performed employing a chemometric approach.

\section{Experimental}

Seventeen biogenic amines: propylamine, dimethylamine, diethylamine, methylamine, tryptamine, cadaverine, spermine, 2-phenylethylamine, tyramine, putrescine, histamine, butylamine, hexylamine, isopentylamine, isobutylamine, spermidine, agmatine, acetonitrile (LC-MS grade), and tosyl chloride $(\geq 99 \%)$ were obtained from Sigma Aldrich (St. Louis, USA). Formic acid (FA) was purchased from Merck (Darmstadt, Germany). Boric acid and sodium hydroxide were purchased from POCH (Gliwice, Poland). Nylon Captiva Econofilters (25 mm diameter, $0.2 \mu \mathrm{m}$ pore size) were purchased from Agilent Technologies (Santa Clara, USA). Ultrapure water was prepared using $\mathrm{HLP}_{5}$ system from Hydrolab (Wiślina, Poland). Borate buffer was prepared by titrating $0.5 \mathrm{M}$ boric acid solution with sodium hydroxide to the required $\mathrm{pH}$ value. Chemical structures of studied biogenic amines are shown in Fig. 4.

\section{Instrumentation}

The HPLC-MS/MS analyses were performed using an Agilent 1200 LC system equipped with binary pump, an online degasser, an autosampler and a thermostated column compartment equipped with a switching valve coupled with an AB Sciex 4000 QTRAP mass spectrometer. Gemini C-18 (150 × $4.6 \mathrm{~mm}, 3 \mu \mathrm{m}$, Phenomenex $)$ column was used for RP-HPLC separation of the derivatives of biogenic amines. The mobile phase consisted of water containing $0.1 \%$ formic acid (component $\mathrm{A}$ ) and acetonitrile containing $0.1 \%$ formic acid (component B). The gradient elution was as follows: $20 \%$ of B for $2 \mathrm{~min}$, then linear increase to $65 \% \mathrm{~B}$ during $15 \mathrm{~min}, 65 \% \mathrm{~B}$ maintained for $3 \mathrm{~min}$, increase from $65 \% \mathrm{~B}$ to $95 \% \mathrm{~B}$ during $3 \mathrm{~min}$ followed by $95 \% \mathrm{~B}$ maintained for $3 \mathrm{~min}$. The last step was conditioning of the column for 3 min with $20 \%$ B. During the first 2 min of a run, the eluate was directed to waste by means of switching valve. The mobile phase flow rate was $1 \mathrm{~cm}^{3} / \mathrm{min}$ and injection volume in this case was $10 \mathrm{~mm}^{3}$. 
Table 4 MRM transition parameters for detection of amine's tosyl derivatives

\begin{tabular}{|c|c|c|c|}
\hline Compound & $\mathrm{MRM}^{\mathrm{a}}$ & Declustering potential/V & Collision energy/V \\
\hline \multirow[t]{2}{*}{ Methylamine } & $186.101 \rightarrow 155$ & 71 & 17 \\
\hline & $186.101 \rightarrow 91$ & 71 & 29 \\
\hline \multirow[t]{2}{*}{ Dimethylamine } & $200.121 \rightarrow 155$ & 76 & 25 \\
\hline & $200.121 \rightarrow 91$ & 76 & 37 \\
\hline \multirow[t]{2}{*}{ Propylamine } & $214.095 \rightarrow 155.10$ & 71 & 23 \\
\hline & $214.095 \rightarrow 91$ & 71 & 37 \\
\hline \multirow[t]{2}{*}{ Butylamine } & $228.144 \rightarrow 155$ & 66 & 23 \\
\hline & $228.144 \rightarrow 91$ & 61 & 37 \\
\hline \multirow[t]{2}{*}{ Isobutylamine } & $228.144 \rightarrow 155$ & 66 & 23 \\
\hline & $228.144 \rightarrow 91$ & 61 & 37 \\
\hline \multirow[t]{2}{*}{ Diethylamine } & $228.144 \rightarrow 155$ & 66 & 23 \\
\hline & $228.144 \rightarrow 91$ & 61 & 37 \\
\hline \multirow[t]{2}{*}{ 2-Phenylethylamine } & $276.127 \rightarrow 105$ & 66 & 23 \\
\hline & $276.127 \rightarrow 77$ & 66 & 69 \\
\hline \multirow[t]{2}{*}{ Isopentylamine } & $242.157 \rightarrow 155$ & 66 & 27 \\
\hline & $242.157 \rightarrow 91$ & 66 & 43 \\
\hline \multirow[t]{2}{*}{ Tryptamine } & $315.060 \rightarrow 144.10$ & 51 & 17 \\
\hline & $315.060 \rightarrow 117$ & 51 & 77 \\
\hline \multirow[t]{2}{*}{ Cadaverine } & $411.10 \rightarrow 240.20$ & 96 & 23 \\
\hline & $411.10 \rightarrow 184.10$ & 96 & 29 \\
\hline \multirow[t]{2}{*}{ Putrescine } & $397.11 \rightarrow 226.30$ & 96 & 23 \\
\hline & $397.11 \rightarrow 155.10$ & 96 & 35 \\
\hline \multirow[t]{2}{*}{ Spermidine } & $608.284 \rightarrow 383.20$ & 91 & 31 \\
\hline & $608.284 \rightarrow 226.30$ & 106 & 37 \\
\hline \multirow[t]{2}{*}{ Spermine } & $819.336 \rightarrow 212.100$ & 126 & 49 \\
\hline & $819.336 \rightarrow 281.100$ & 126 & 51 \\
\hline \multirow[t]{2}{*}{ Tyramine } & $446.172 \rightarrow 275.100$ & 101 & 21 \\
\hline & $446.172 \rightarrow 155.000$ & 101 & 35 \\
\hline \multirow[t]{2}{*}{ Histamine } & $420.095 \rightarrow 109.000$ & 81 & 35 \\
\hline & $420.095 \rightarrow 91.000$ & 81 & 67 \\
\hline \multirow[t]{2}{*}{ Hexylamine } & $256.190 \rightarrow 155.100$ & 71 & 27 \\
\hline & $256.190 \rightarrow 91.000$ & 71 & 41 \\
\hline \multirow[t]{2}{*}{ Agmatine } & $439.218 \rightarrow 155.200$ & 121 & 37 \\
\hline & $439.218 \rightarrow 91.000$ & 121 & 71 \\
\hline
\end{tabular}

Condition of ESI source: Source temperature $500{ }^{\circ} \mathrm{C}$, nebulizer gas 45 psi, heater gas 30 psi, curtain gas 20 psi, capillary voltage $5000 \mathrm{~V}$

Fig. 5 Diagram illustrating derivatization of wine and beer samples

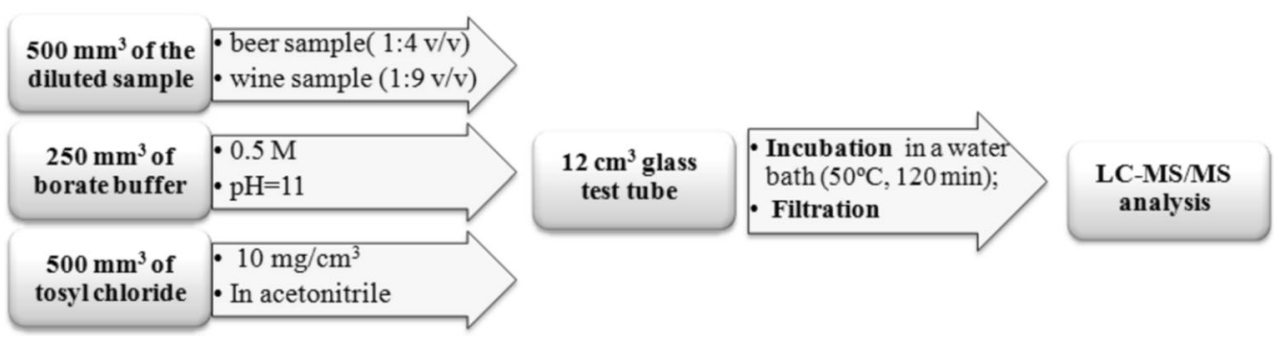


The column temperature throughout the separation process was kept at $40{ }^{\circ} \mathrm{C}$. The ESI source was operated in positive ion mode with the following conditions: the source temperature was set at $500{ }^{\circ} \mathrm{C}$, ion spray voltage was set at $5000 \mathrm{~V}$, nebulizer gas was set at $45 \mathrm{psi}$, and heater gas and curtain gas were set 30 and 20 psi, respectively. Source and MS parameters are shown in Table 4. To acquire chromatograms and control instrumentation, Analyst Software version 1.5.2 (AB Sciex, CA, USA) was used.

\section{Beer and wine sample collection and preparation}

Twenty-eight of sample beers (type of lager) differing in place of production and the alcohol content and eighteen samples of wine differing in the main material (grape, black currant, plum, apple, chokeberry, quince, raspberry, white grape, strawberry) were purchased at local supermarkets.

All beers and wines were analyzed within one day from purchase. In case of beer, samples were degassed in an ultrasonic bath for $10 \mathrm{~min}$ and diluted $(1+4, \mathrm{v} / \mathrm{v})$ with ultrapure water, while in case of wine, degassing step was omitted and the wine samples were diluted $(1+9$, v/v $)$ with ultrapure water.

To perform derivatization, $500 \mathrm{~mm}^{3}$ of diluted beer or wine sample was transferred to a $12 \mathrm{~cm}^{3}$ glass test tube and mixed with $250 \mathrm{~mm}^{3}$ of borate buffer $(0.5 \mathrm{M}, \mathrm{pH}=11)$ and $500 \mathrm{~mm}^{3}$ of tosyl chloride solution $\left(10 \mathrm{mg} / \mathrm{cm}^{3}\right.$, in acetonitrile). After mixing, samples were incubated for $120 \mathrm{~min}$ at $50^{\circ} \mathrm{C}$ in water bath. Finally, the samples were filtered through a $0.2 \mu \mathrm{m}$ nylon filter (Agilent Technologies, Santa Clara, CA, USA) and injected into chromatographic system. Overall way for the preparation of beer and wine samples is shown in Fig. 5.

\section{Calibration curves}

Stock solutions $\left(1 \mathrm{mg} / \mathrm{cm}^{3}\right)$ of each biogenic amine were prepared in $0.1 \mathrm{M} \mathrm{HCl}$. Then, in order to produce the

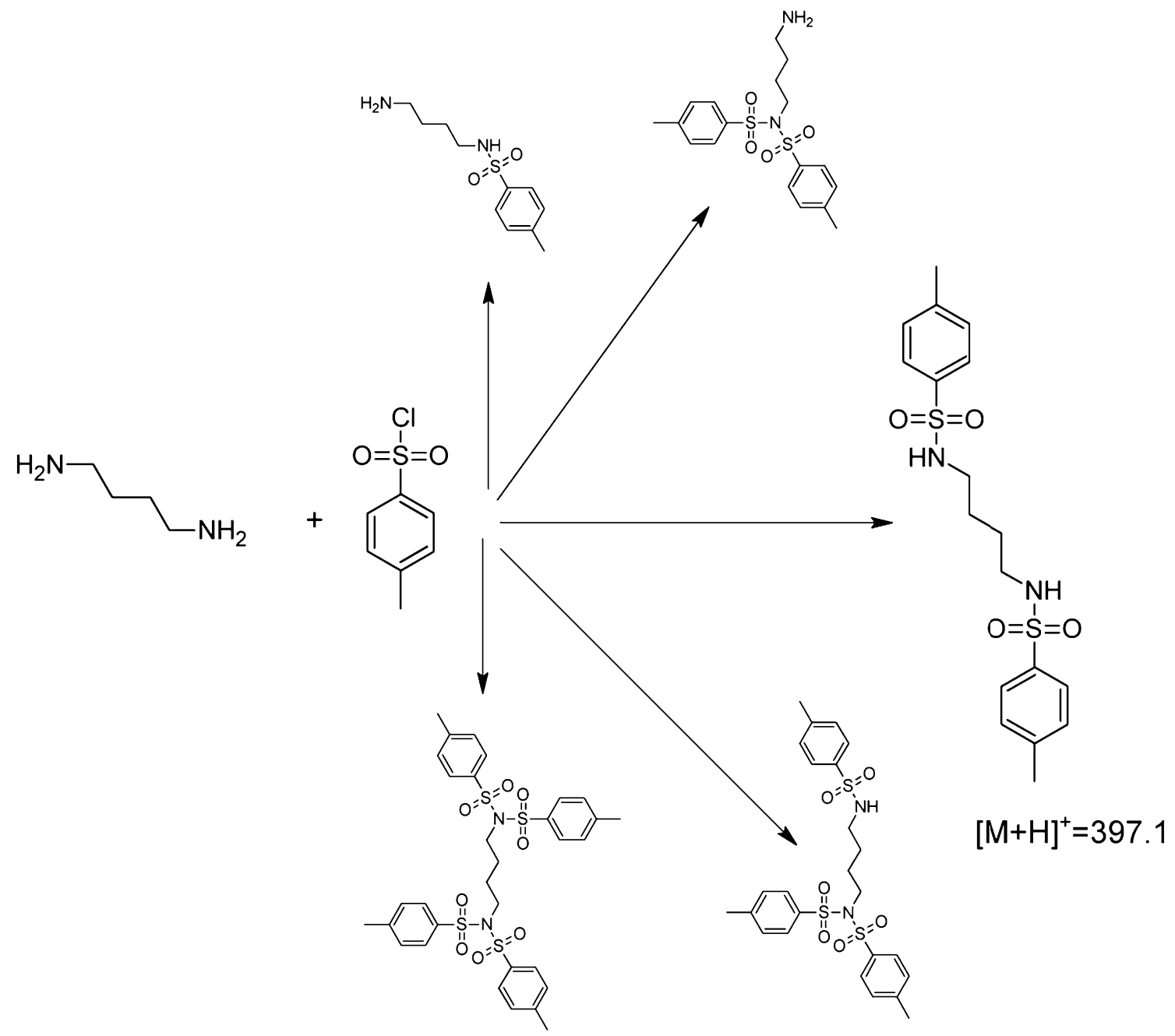

Fig. 6 Formation of putrescine's tosyl derivatives (tentative, favored structure enlarged) 
Fig. 7 Estimation of matrix effects. Slopes of regression lines calculated after spiking the samples of wine and beer with mixture of BA's standards were normalized against the water sample

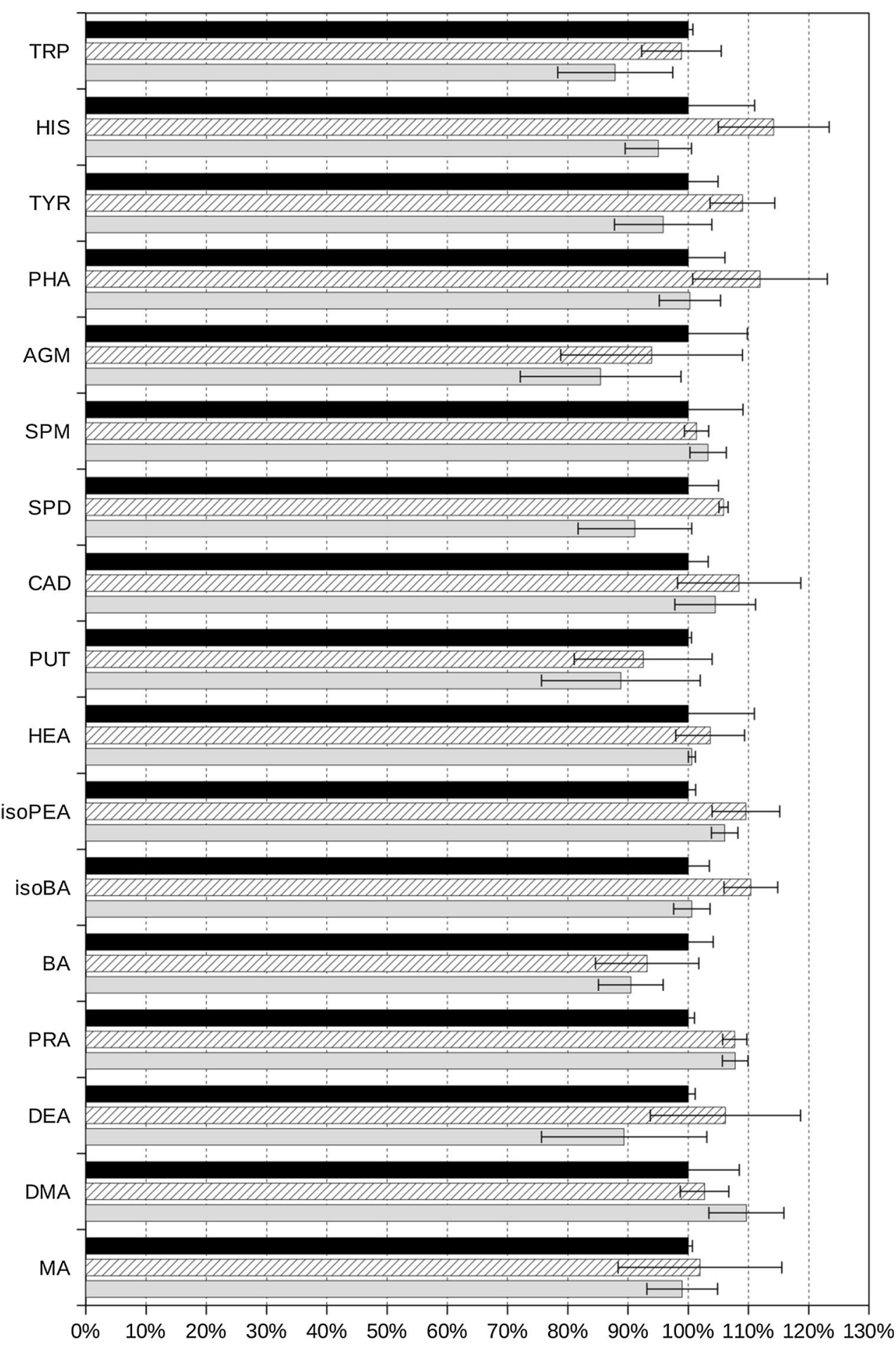

$\square$ beer $\square$ wine $\square$ water standard mix, relevant aliquots of each of seventeen solutions were introduced into the $25 \mathrm{~cm}^{3}$ volumetric flask and made up to the mark with acetonitrile: $0.1 \mathrm{M} \mathrm{HCl}(3+7$, $\mathrm{v} / \mathrm{v})$ mixture. The concentrations of each biogenic amine have been adjusted on the basis of preliminary analyses of the samples. Standard mix prepared in this way has been used to prepare the calibration curves. Six points (each point in triplicate) calibration curves were prepared by diluting variable aliquots of the standard mix with acetonitrile: $0.1 \mathrm{M} \mathrm{HCl}(3+7, \mathrm{v} / \mathrm{v})$ mixture. Thereafter, 
$100 \mathrm{~mm}^{3}$ of each calibration solution was mixed with $400 \mathrm{~mm}^{3}$ of ultrapure water and subjected to the same derivatization procedure as beer and wine samples.

One must be aware that the derivatization reaction may lead to the formation of different, multiply tosylated amine derivatives. Formation of these derivatives is influenced by different factors, steric effect being probably the most important one. During the development of the method described here, every single amine has been subjected to the derivatization and the product giving the most intense spectrometric peak has been selected. It turned out that substitution of single hydrogen, bound to the primary (or secondary) nitrogen atom proceeds fastest in most cases. The derivatization scheme (with putrescine as an example) is presented in Fig. 6.

\section{Matrix effects}

The impact of sample matrix on derivatization yield was estimated using standard addition method. Three kinds of samples were derivatized: water, wine, and beer. The samples (each in triplicate) were spiked at three different concentration levels with the same amount of standard mixture of biogenic amines. The average peak area corresponding to each analyte was plotted against the added amount of the analyte. Linear regression lines were calculated and their slopes compared. The results of this comparison are shown in Fig. 7. In general, it seems that wine matrix slightly increases derivatisation yield while beer matrix acts just opposite. The observed effects are more or less randomly visible among different biogenic amines derivatives (characterized by different retention times); therefore, in our opinion the core cause of this enhancement or inhibition lies in the derivatization reactions. The exact mechanism is unknown at the time of writing but certainly it is worth further studies.
Open Access This article is distributed under the terms of the Creative Commons Attribution 4.0 International License (http:// creativecommons.org/licenses/by/4.0/), which permits unrestricted use, distribution, and reproduction in any medium, provided you give appropriate credit to the original author(s) and the source, provide a link to the Creative Commons license, and indicate if changes were made.

\section{References}

1. Önal A (2007) Food Chem 103:1475

2. Płotka-Wasylka JM, Morrison C, Biziuk M, Namieśnik J (2015) Chem Rev 115:4693

3. Kalač P, Hlavată V, Kŕižek M (1997) Food Chem 58:209

4. Vinci G, Antonelli ML (2002) Food Control 13:519

5. Innocente N, Biasutti M, Padovese M, Moret S (2007) Food Chem 101:1285

6. Loret S, Deloyer P, Dandrifosse G (2005) Food Chem 89:519

7. Shalaby AR (1996) Food Res Int 29:675

8. Draisci R, Volpe G, Lucentini L, Cecilia A, Federico R, Palleschi G (1998) Food Chem 62:225

9. Ordóñez JL, Troncoso AM, Garćia-Parrilla MC, Callejón RM (2016) Anal Chim Acta 939:10

10. EFSA Panel on Biological Hazards (BIOHAZ) (2011) EFSA J 9:2393

11. Smit AY, du Toit WJ, du Toit M (2008) S Afr J Enol Vitic 29:109

12. Galgano F, Caruso M, Perretti G, Favati F (2011) Eur Food Res Technol 232:889

13. Yañez L, Saavedra J, Martinez C, Córdova A, Ganga MA (2012) J Food Sci 77:143

14. Romero R, Sanchez-Vinas M, Gazquez D, Bagur MG (2002) J Agric Food Chem 50:4713

15. Proestos Ch, Loukatos P, Komaitis M (2008) Food Chem 106:1218

16. Callejón RM, Troncoso AM, Morales ML (2010) Talanta 81:1143

17. Zhao QX, Xu J, Xue ChH, Sheng WJ, Gao RCh, Xue Y, Li ZJ (2007) J Agric Food Chem 55:3083

18. Dziarkowska K, Jönsson JÁ, Wieczorek PP (2008) Anal Chim Acta 606:184

19. Jastrzębska A, Piasta A, Kowalska S, Krzemiński M, Szłyk E (2016) J Food Compos Anal 48:111

20. Pulido MI, Hernández-Jover T, Mariné-Font A, Vidal-Carou MC (1996) J Agric Food Chem 44:3159 